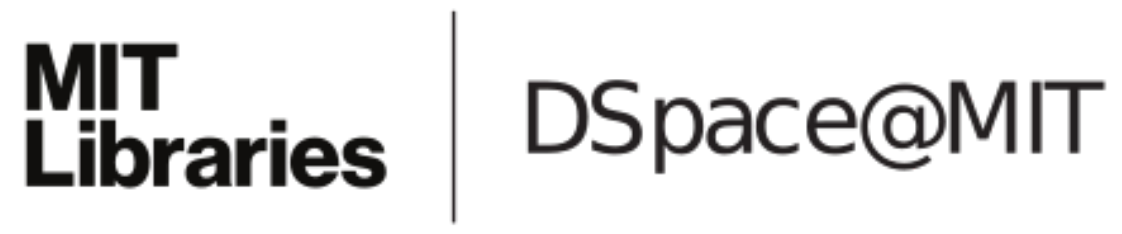

\author{
MIT Open Access Articles
}

Implanted Nanosensors in Marine Organisms for Physiological Biologging: Design, Feasibility, and Species Variability

The MIT Faculty has made this article openly available. Please share how this access benefits you. Your story matters.

As Published: $10.1021 /$ ACSSENSORS.8B00538

Publisher: American Chemical Society (ACS)

Persistent URL: https://hdl.handle.net/1721.1/134899

Version: Author's final manuscript: final author's manuscript post peer review, without publisher's formatting or copy editing

Terms of Use: Article is made available in accordance with the publisher's policy and may be subject to US copyright law. Please refer to the publisher's site for terms of use. 


\section{Implanted Nanosensors in Marine Organisms for Physiological Biologging: Design, Feasibility, and Species Variability}

3 Michael A. Lee, ${ }^{\dagger}$ Freddy T. Nguyen, ${ }^{\dagger}$ Kathleen Scott, ${ }^{\dagger}$ Nathan Y.L. Chan, ${ }^{\S}$ Naveed Ali Bakh, ${ }^{\dagger}$ ${ }_{4}$ Kelvin K. Jones ${ }^{\dagger}$ Crystal Pham, ${ }^{\dagger}$ Pablo Garcia-Salinas," Daniel Garcia-Parraga, Andreas Fahlman, ${ }_{5}$ Vicente Marco, $"$ Volodymyr B. Koman, ${ }^{\dagger}$ Ronnie J. Oliver, ${ }^{\dagger}$ Lloyd W. Hopkins, ${ }^{\perp}$ Consuelo Rubio," ${ }_{6}$ Rory P. Wilson, ${ }^{\perp}$ Mark G. Meekan, ${ }^{\#}$ Carlos M. Duarte, ${ }^{\mathbb{I}}{ }^{\dagger}$ and Michael S. Strano* ${ }^{\dagger}$ (1)

$7{ }^{\dagger}$ Department of Chemical Engineering and ${ }^{\S}$ Division of Comparative Medicine, Massachusetts Institute of Technology, Cambridge, 8 Massachusetts 02139, United States

9 *Office of Animal Resources, University of Iowa, Iowa City, Iowa 52242, United States

10 "Fundación Oceanogràfic de la Comunitat Valenciana, Research Department, Ciudad de las Artes y las Ciencias, 46013 Valencia, 11 Spain

$12{ }^{\perp}$ Biosciences, College of Science, Swansea University, Singleton Park, Swansea SA2 8PP, United Kingdom

13 \#Australian Institute of Marine Science, the Indian Ocean Marine Research Centre (IOMRC), University of Western Australia

14 Oceans Institute, 35 Stirling Highway, Crawley, Western Australia 6009, Australia

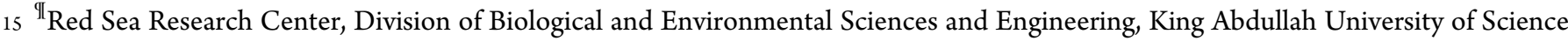
16 and Technology, Thuwal 23955-6900, Saudi Arabia

46 $\mathrm{T}$ recent decades, the biologging community has attached ${ }_{47}$ various types of sensors to animals to characterize animal 48 behavior in the context of their environments. ${ }^{1}$ These studies
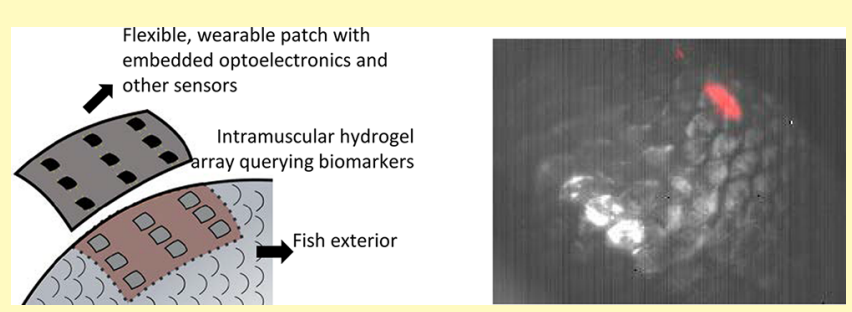
data collection constraints of implantable, near-infrared fluorescent nanosensors based on DNA-wrapped single-wall carbon nanotubes (SWNT) embedded within a biocompatible poly(ethylene glycol) diacrylate (PEGDA) hydrogel. These sensors are enabled by Corona Phase Molecular Recognition (CoPhMoRe) to provide selective chemical detection for marine organism biologging. Riboflavin, a key nutrient in oxidative phosphorylation, is utilized as a model analyte in in vitro and ex vivo tissue measurements. Nine species of bony fish, sharks, eels, and turtles were utilized on site at Oceanogràfic in Valencia, Spain to investigate sensor design parameters, including implantation depth, sensor imaging and detection limits, fluence, and stability, as well as acute and long-term biocompatibility. Hydrogels were implanted subcutaneously and imaged using a customized, field-portable Raspberry Pi camera system. Hydrogels could be detected up to depths of $7 \mathrm{~mm}$ in the skin and muscle tissue of deceased teleost fish (Sparus aurata and Stenotomus chrysops) and a deceased catshark (Galeus melastomus). The effects of tissue heterogeneity on hydrogel delivery and fluorescence visibility were explored, with darker tissues masking hydrogel fluorescence. Hydrogels were implanted into a living eastern river cooter (Pseudemys concinna), a European eel (Anguilla anguilla), and a second species of catshark (Scyliorhinus stellaris). The animals displayed no observable changes in movement and feeding patterns. Imaging by high-resolution ultrasound indicated no changes in tissue structure in the eel and catshark. In the turtle, some tissue reaction was detected upon dissection and histopathology. Analysis of movement patterns in sarasa comet goldfish (Carassius auratus) indicated that the hydrogel implants did not affect swimming patterns. Taken together, these results indicate that this implantable form factor is a promising technique for biologging using aquatic vertebrates with further development. Future work will tune the sensor detection range to the physiological range of riboflavin, develop strategies to normalize sensor signal to account for the optical heterogeneity of animal tissues, and design a flexible, wearable device incorporating optoelectronic components that will enable sensor measurements in moving animals. This work advances the application of nanosensors to organisms beyond the commonly used rodent and zebrafish models and is an important step toward the physiological biologging of aquatic organisms.

KEYWORDS: SWNT, in vivo, biologging, aquatic organisms, hydrogel, sensor

Received: June 22, 2018

Accepted: November 20, 2018
ABSTRACT: In recent decades, biologists have sought to tag animals with various sensors to study aspects of their behavior otherwise inaccessible from controlled laboratory experiments. Despite this, chemical information, both environmental and physiological, remains challenging to collect despite its tremendous potential to elucidate a wide range of animal behaviors. In this work, we explore the design, feasibility, and 


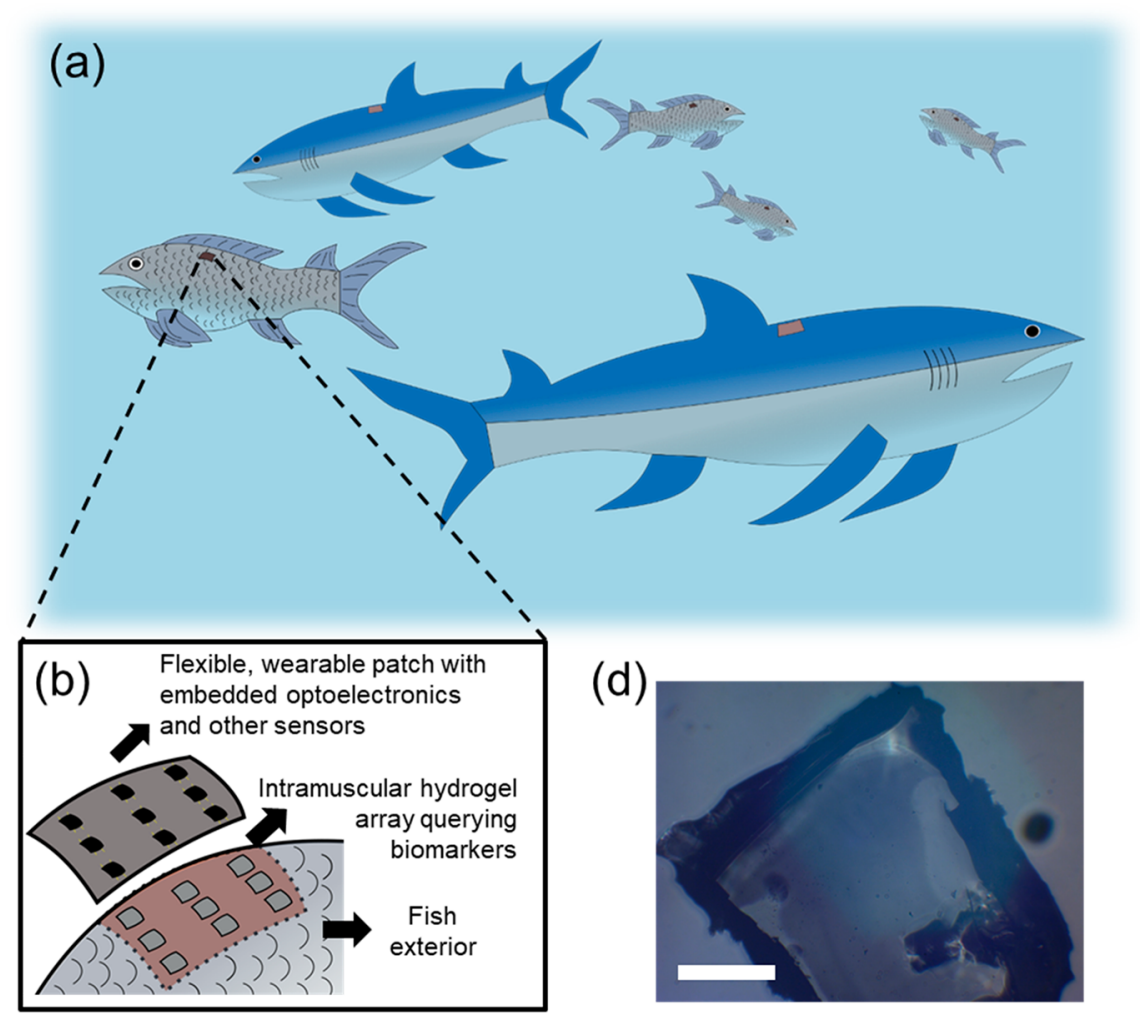

(c)

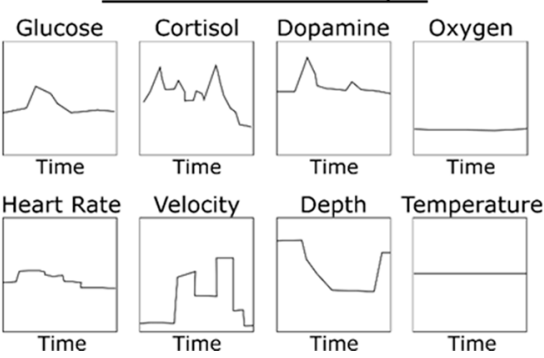

(e)

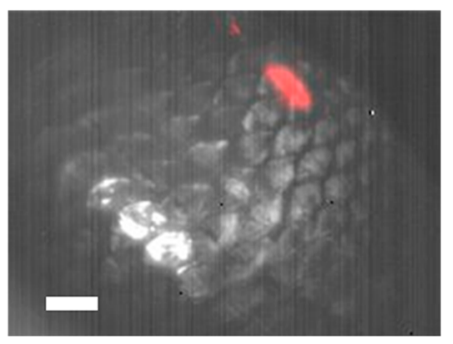

Figure 1. Vision for the future application of CoPhMoRe sensors to physiological biologging of marine organisms. (a) Animals of various sizes and ecological niches tagged with minimally invasive sensors collecting multivariate data sets continuously. (b) Theoretical design of a future biologging system. Hydrogel implants, encapsulating nanoparticles engineered to modulate their fluorescence in response to the local concentration of specific bioanalytes, are injected at a fixed depth in the intramuscular space, where they query biological fluid. Atop the fish's exterior is a flexible, wearable patch that contains embedded optoelectronics to excite and collect hydrogel fluorescence. The elastomer protects the electronic components from the surrounding aquatic environment, as well as conforming to the animal's movements. The device also incorporates other sensors to track animal movement and environmental conditions. The work herein describes the development of the hydrogel component of this theoretical device. (c) Theoretical data output of envisioned device. The device collects biochemical information and other animal-derived and environmental parameters such as velocity, depth, temperature, etc. (d) Visible image of SWNT-gels ( scale $=0.5 \mathrm{~mm}$ ). (e) Overlay of bright field image of sarasa comet goldfish ( Carassius auratus) and fluorescence image of implanted hydrogel (scale $=10 \mathrm{~mm}$ ).

49 have produced key insights into a wide range of ecological 50 phenomena, including the metabolic energy balance, ${ }^{2}$ preda51 tor-prey relationships, ${ }^{3}$ the ecological effects of climate 52 change, ${ }^{4}$ the impact of human activity on animals, ${ }^{5}$ and other 53 behaviors related to feeding, ${ }^{6}$ migration, ${ }^{7}$ and reproduction. ${ }^{8}$ 54 However, deployed sensors have largely been limited to 55 environmental parameter sensors (temperature, pressure, and 56 salinity), movement and location sensors (accelerometers and 57 GPS), and vital sign sensors, such as heart rate monitors. ${ }^{2}$ 58 Notably missing from these tools are chemical sensors. These 59 may be outward-facing, measuring analytes in the local 60 environment around the animal, or inward-facing, measuring 61 biochemical signaling pathways within the animal. The advent of 62 novel technologies capable of real-time, continuous chemical sensing, such as those enabled by Corona Phase Molecular 63 Recognition (CoPhMoRe), may enable access to this 64 information and thereby significantly advance biologging 65 studies. ${ }^{9}$ Herein, we explore, for the first time, several design 66 and operation issues associated with implantable sensors of this 67 type for biologging applications, using near-infrared (nIR) 68 fluorescent carbon nanotube sensors as a model for marine 69 organisms to address aspects of feasibility. For this study and 70 purpose, we have assembled a unique team of marine biologists, 71 sensor developers, and engineers to address this challenge, as 72 coauthors of this study.

Recent developments in in vivo sensing technologies offer 74 tremendous opportunities for biologgers to probe the chemical 75 network underpinning animal behaviors. As many excellent 76 
77 reviews have reported, in vivo sensors operating in several 78 modalities-including optical and electrochemical-have been 79 developed to measure a variety of biomarkers, including ions, 80 reactive oxygen species, redox active molecules, oxygen, metals, 81 and macromolecules, among many others. ${ }^{10-18}$ Recently, Sun et 82 al. measured glucose in mice using oxygen-sensitive polymer 83 dots and a smartphone. ${ }^{19}$ Measurements of hypochlorous acid 84 and $\mathrm{pH}$ have been performed in zebrafish and their embryos. ${ }^{20,21}$ 85 Ferreira et al. modified carbon fiber microelectrodes and 86 simultaneously measured ascorbate and glutamate in the 87 hippocampi of anesthetized rats. ${ }^{22}$ Despite these advances, the 88 continuous glucose monitor remains one of the few technologies 89 to be adopted due to stringent analytical and biocompatibility 90 requirements for sensor integrity in in vivo environments. ${ }^{13,14}$ 91 Although biologically derived units such as antibodies, aptamers, 92 and enzymes have traditionally been used for chemical 93 sensing, ${ }^{23}$ they may lose their capability for molecular 94 recognition when conjugated to other sensor components and 95 may also suffer from limited thermal and chemical stability, 96 restricting their use in vivo to short periods of time. ${ }^{24}$

97 Synthetic sensing approaches have overcome some of these 98 disadvantages. Our group has developed Corona Phase 99 Molecular Recognition (CoPhMoRe), which uses a nIR 100 fluorescent nanoparticle that acts as both the molecular 101 recognition unit and the reporter of binding events. ${ }^{9}$ An 102 amphiphilic polymer or surfactant adsorbs onto singly dispersed 103 single-wall carbon nanotubes (SWNT) via hydrophobic 104 interactions. The hydrophilic groups on the polymer provide 105 the dispersion colloidal stability in aqueous solutions, where a 106 majority of bioanalytes exist. The conformation of the adsorbed 107 phase, or the corona, modulates analyte binding to the 108 nanoparticle and provides selectivity. Upon analyte binding, 109 the fluorescence intensity and/or peak wavelength may change. 110 To date, CoPhMoRe sensors have been fabricated for a variety 111 of molecules, including nitric oxide, ${ }^{25}$ hydrogen peroxide, ${ }^{26}$ 112 riboflavin, L-thyroxine, estradiol, ${ }^{9}$ dopamine, ${ }^{27,28}$ fibrinogen, ${ }^{24}$ 113 and insulin. ${ }^{29}$ The nitric oxide sensor has been demonstrated in 114 vivo and shown to have a fluorescence stability of over 400 days 115 within the body of a mouse. ${ }^{30}$

116 The challenge facing researchers is to now incorporate these 117 new types of physiological sensors into biologging devices. ${ }^{31}$ In 118 the past, the biologging community has traditionally focused on 119 sensors that describe the behaviors, external environments, and 120 location of animals. Accelerometers, depth, and temperature 121 sensors and Argos satellite-linked and GPS tags have been 122 central to this task. ${ }^{32}$ For example, using accelerometers, Wilson 123 et al. studied the significance of neck length in swimming and 124 foraging behaviors in Imperial cormorants and Megallanic 125 penguins, ${ }^{33}$ Hays et al. used records from satellite tagging of 126 thousands of sea turtles to compare their migration distances 127 with those of other similarly sized marine animals, ${ }^{7}$ and Meekan 128 et al. used a combination of an accelerometer, magnetometer, 129 GPS, and depth sensors to study the energy efficiency of whale 130 shark movement patterns. ${ }^{34}$

131 The combination of sensors that collect data sets of 132 movement, location, and relevant biochemical parameters 133 (such as glucose, dopamine, and cortisol) ${ }^{28,29,35}$ into biologging 134 tags potentially offers unprecedented insights into the behavior, 135 ecology, and condition of animals. To date, physiological data in 136 biologging tags has mostly been obtained from electromyogram 137 (EMG) and heart rate sensors. ${ }^{2}$ Although there have been a few 138 examples of bioanalyte measurements in extracted blood, ${ }^{36-38}$ 139 the measurement of biomarkers in sampled fluid ex vivo offers limited information and may introduce artifacts due to the 140 capture and restraint of the animal. ${ }^{35}$ CoPhMoRe sensors 141 incorporated into animal-borne sensor tags have the potential to 142 transform biologging studies by giving researchers continuous 143 and real-time access to biomarkers reflecting the condition of 144 free-living animals (Figure 1). ${ }^{39,40}$

145

In this work, as a model sensor implant, we use DNA-wrapped 146 SWNT that we have fabricated and encapsulated into a 147 biocompatible poly(ethylene glycol diacrylate) (PEGDA) 148 hydrogel and calibrated against riboflavin, an essential nutrient 149 involved in oxidative phosphorylation. ${ }^{41}$ In vitro characterization 150 and experiments with two species of marine organisms were 151 performed at MIT, whereas experiments with an additional 152 seven species were performed at Oceanogràfic in Valencia, Spain 153 from January 30 to February 1, 2018. The implants were 154 delivered via trocar to both recently deceased and living animals. 155 The hydrogel detection limit with injection depth was 156 determined, and the effects of tissue heterogeneity on 157 fluorescence detection were explored. The three living animals 158 showed no external signs of adverse health or behavioral changes 159 one month after implantation. However, in the case of the turtle, 160 some tissue reaction was detected upon dissection and 161 histopathology. At MIT, analysis of goldfish swimming patterns 162 indicated that the hydrogel implants do not impair animal 163 movement. All together, these data indicate the feasibility of 164 using CoPhMoRe sensors for marine organism biologging with 165 further improvements to sensor detection limits, normalization 166 of sensor signal to account for individual tissue optical 167 properties, and wearable fluorescence device design.

\section{METHODS AND MATERIALS}

169

Materials. (6,5)-Enriched SWNTs produced by the CoMoCAT 170 process (lot \# MKBZ1159 V) were purchased from Sigma-Aldrich. 171 Single-stranded $(\mathrm{AC})_{15}$ was purchased from Integrated DNA 172 Technologies, while PEGDA $\left(M_{\mathrm{n}}=8000\right)$ was purchased from Alfa 173 Aesar. Unless otherwise noted, other reagents were purchased from 174 Sigma-Aldrich.

Sensor Fabrication. SWNT $(1 \mathrm{mg} / \mathrm{mL})$ and ss $(\mathrm{AC})_{15}(2 \mathrm{mg} / \mathrm{mL}) 176$ were mixed in $2 \mathrm{~mL}$ of $100 \mathrm{mM}$ sodium chloride. The mixture was bath 177 sonicated for $10 \mathrm{~min}$, followed by sonication with a $3 \mathrm{~mm}$ probe at $4 \mathrm{~W} 178$ for $20 \mathrm{~min}$ (QSonica). The suspension was centrifuged at 32,000 rcf for 179 $3 \mathrm{~h}$, and the top $80 \%$ of the supernatant was collected for further use. 180 Free DNA was removed using $100 \mathrm{kDa}$ MWCO centrifugal filters 181 (Merck Millipore) with 5 volumetric replacements with $1 \times$ phosphate 182 buffered saline (PBS). UV-vis-NIR absorption spectra were collected 183 to verify successful suspension. The SWNT mass concentration was 184 calculated using the absorption value at $632 \mathrm{~nm}$. 185

ssDNA-SWNT $(0.1 \mathrm{mg} / \mathrm{mL})$, PEGDA (100 mg/mL), and 2- 186 hydroxy-4'-(2-hydroxyethoxy)-2-methylpropiophenone (0.175 mg/ 187 $\mathrm{mL}$ ) were mixed in $1 \times$ PBS, cast into glass molds, and incubated for 188 30 min under a nitrogen atmosphere. The samples were then 189 illuminated under $365 \mathrm{~nm}$ ultraviolet radiation (UVP Blak-Ray XX- 190 $15 \mathrm{BLB}, 15 \mathrm{~W}$ ) for $60 \mathrm{~min}$. The hydrogels were removed from the molds 191 and incubated in excess $1 \times$ PBS for $48 \mathrm{~h}$ to remove unreacted 192 monomers and unencapsulated SWNT. The hydrogels were then 193 incubated in fresh $1 \times$ PBS until further use.

In Vitro Characterization. UV-vis-NIR absorption spectra were 195 measured for both solution phase and hydrogel encapsulated ssDNA- 196 SWNT (Shimadzu UV-3101PC). Fluorescence spectra were measured 197 in a custom-built NIR microscope. Samples were illuminated using a 198 $785 \mathrm{~nm}$ photodiode laser (B\&W Tek. Inc.) and imaged using a Zeiss 199 AxioVision inverted microscope with appropriate optical filters. The 200 fluorescence was passed through a Princeton Instruments Acton 201 SP2500 spectrometer and measured using a liquid nitrogen cooled 202 Princeton Instruments InGaAs 1D detector. 

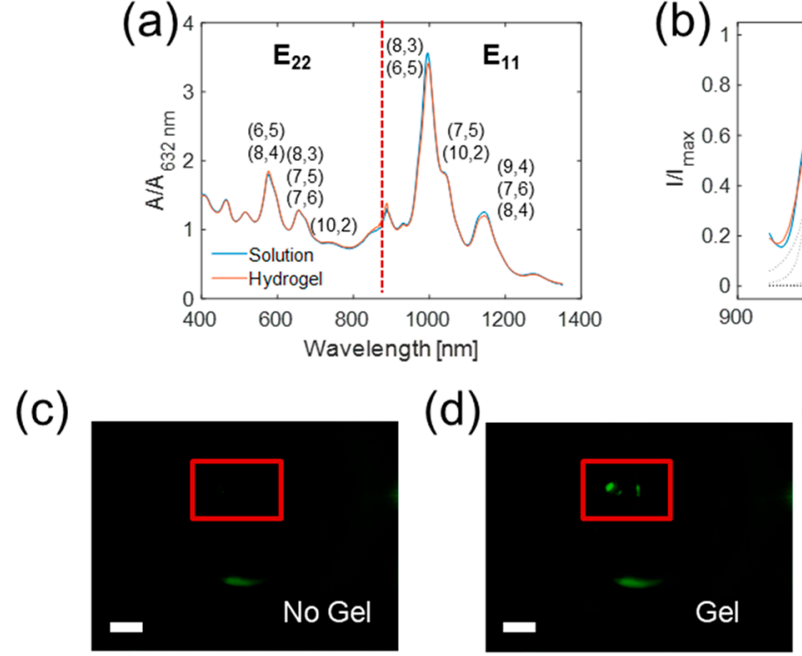

(f)

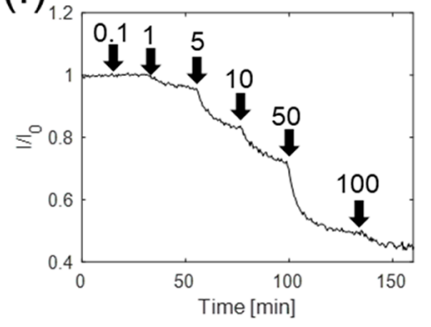

$(\mathrm{g})$

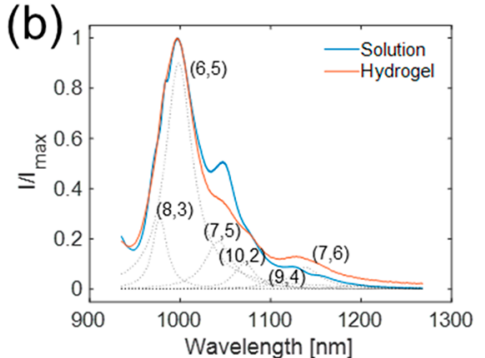

(e)

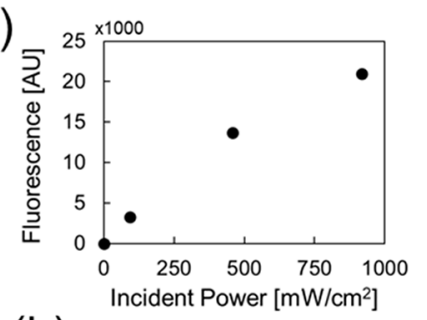

(h)
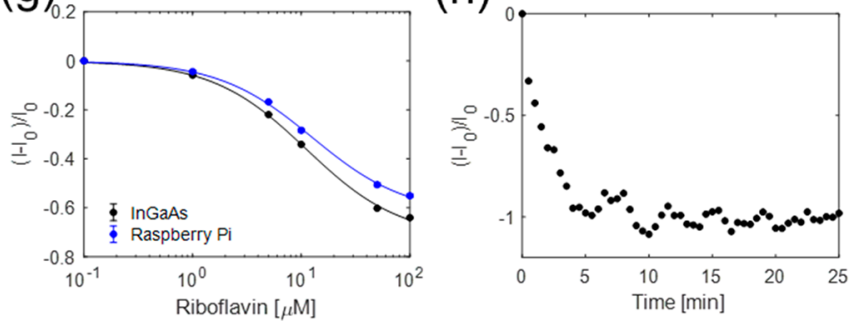

Figure 2. In vitro and ex vivo sensor characterization. (a) Normalized UV-vis-NIR absorption spectra and (b) fluorescence emission spectrum at 785 $\mathrm{nm}$ excitation of of ss(AC) 15 -wrapped $(6,5)$ CoMoCAT SWNT. Spectra were measured for solution phase SWNT and SWNT-gels. The absorption spectrum shows both the excitation $\left(E_{22}\right)$ and fluorescence emission peaks $\left(E_{11}\right)$ for the corresponding SWNT chiralities given in parentheses. The fluorescence spectrum was decomposed into individual peaks corresponding to the labeled SWNT chiralities. (c,d) Images taken with Raspberry Pi imaging setup (c) without and (d) with a SWNT-gel. (e) Hydrogel fluorescence increased with larger incident excitation power. (f) Fluorescence decreased with stepwise increases in riboflavin concentration between 1 to $100 \mu \mathrm{M}$, as measured by a Raspberry Pi camera. (g) Riboflavin calibration curves obtained with an InGaAs camera and the Raspberry Pi camera show good agreement. (h) SWNT-gel response to bolus injection of $100 \mu \mathrm{M}$ riboflavin while placed $1 \mathrm{~mm}$ deep into ex vivo tissue sample of Stenotomus chrysops. The fluorescence decreased below the limit of detection of the Raspberry Pi camera.

204 Riboflavin was used as a model analyte to test hydrogel chemical 205 sensitivity in vitro and ex vivo. Hydrogels were cut into $5 \times 5 \times 1 \mathrm{~mm}^{3}$ 206 sections and placed inside perfusion channels (ibidi $\mu$-Slide III 3D 207 Perfusion). Hydrogel fluorescence was monitored while varying the 208 concentration of riboflavin in $1 \times \mathrm{PBS}$ between $0-100 \mu \mathrm{M}$ at a flow rate 209 of $0.3 \mathrm{~mL} / \mathrm{min}$. Fluorescence images were taken using a liquid nitrogen 210 cooled Princeton Instruments InGaAs $2 \mathrm{D}$ detector. These measure211 ments were also performed on a $5 \times 5 \times 2 \mathrm{~mm}^{3}$ section of hydrogel 212 placed $1 \mathrm{~mm}$ below the surface of skin and muscle tissue of Stenotomus 213 chrysops. A $500 \mu \mathrm{L}$ bolus of $100 \mu \mathrm{M}$ riboflavin was introduced atop of 214 the hydrogel.

215 In Vivo Implantation. All procedures described below were 216 approved by the animal ethics committee of the Fundación Ocean217 ogràfic de la Comunitat Valenciana and performed at Oceanogràfic over 218 the duration of the experiments.

219 Prior to implantation, hydrogels were illuminated by UV light for 15 $220 \mathrm{~min}$ and handled in a biological hood (Telstar AV-100) thereafter to 221 ensure sterility. Hydrogels were cut to a $1 \times 5 \times 1 \mathrm{~mm}^{3}$ block and loaded 222 into 12 gauge transponder needles from which the microchips were 223 removed (Avid Suds Monoject).

224 The implantation procedure varied depending on the target 225 organism. In the case of deceased animals, all animals were injected 226 without further treatment of the skin. Hydrogels were placed at the 227 desired location and penetration depth by using the needle length and 228 angle of insertion as a guide.

229 A live European eel (Anguilla anguilla) was anesthetized prior to 230 injection by submersion in a $70 \mathrm{mg} / \mathrm{L}$ benzocaine solution. When the 231 eel was nonresponsive, the injection site on the dorsal side was washed with sterile saline, and the hydrogel was injected. The eel was moved to 232 new water and allowed to recover prior to further handling. 233

A live eastern river cooter (Pseudemys concinna) and catshark ( 234 Scyliorhinus stellaris) were restrained by animal care personnel for 235 hydrogel implantations. The skin of the shark was washed with sterile 236 saline, whereas the skin of the turtle was disinfected with iodopovidone. 237 The hydrogel was injected subcutaneously in the dorsal area of the 238 shark at the level of the second dorsal fin and in the dorsal part of the 239 cranial tram of the turtle's neck.

240

After implantation, the animals were monitored for 2 months to 241 determine tolerance to the implants and changes in swimming and 242 feeding behavior. High-resolution ultrasound images of the implanta- 243 tion site were used to noninvasively study the impacts of implantation 244 on tissues. After one month, the turtle was euthanized (for reasons not 245 related to this study), allowing biopsies of the implantation site to be 246 collected for histopathology.

Imaging Using Raspberry Pi. The imaging system consisted of a 248 Raspberry Pi 3 (Adafruit) with a 5 MP camera with the IR filter 249 removed (SainSmart). The camera was placed inside of a 1 in. lens tube. 250 The camera was used without further modification when taking 251 brightfield images. The Picamera software package was used to control 252 the camera.

When taking fluorescence images, the hydrogels were illuminated 254 with a $200 \mathrm{~mW} 561 \mathrm{~nm}$ laser (Opto Engine LLC) passing through a 255 collimator. Fluorescence passed through a 900 long-pass filter prior to 256 collection by the camera. Fluorescence was quantified by taking two 257 images before and after hydrogel placement and calculating the 258 difference in gray value in the region of interest. For all images, the 259 
(a)

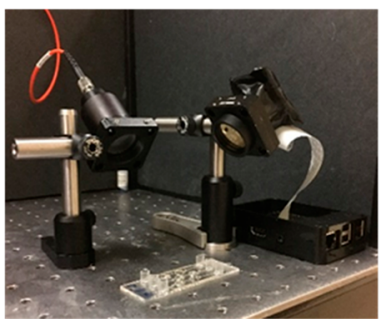

(b)

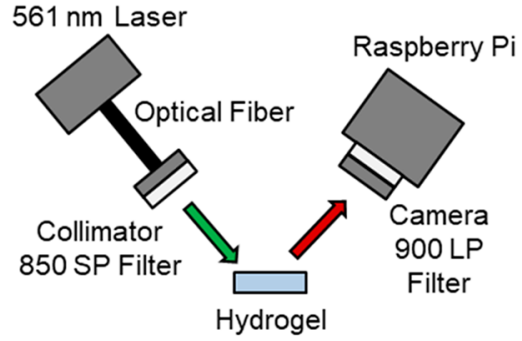

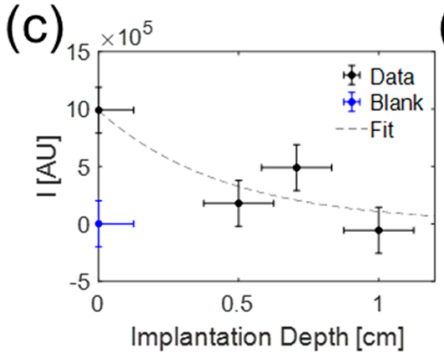

(d)

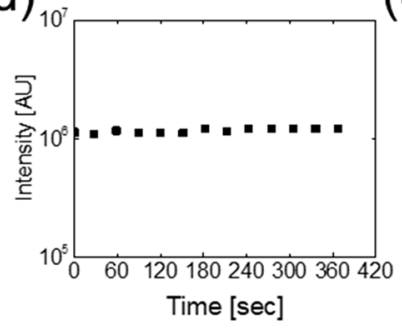

(e)

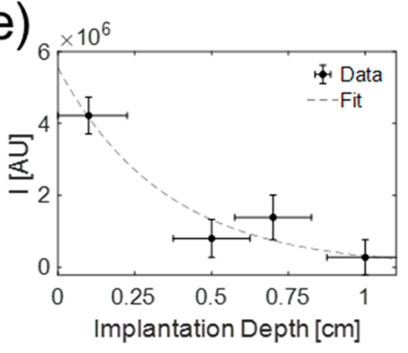

(f)
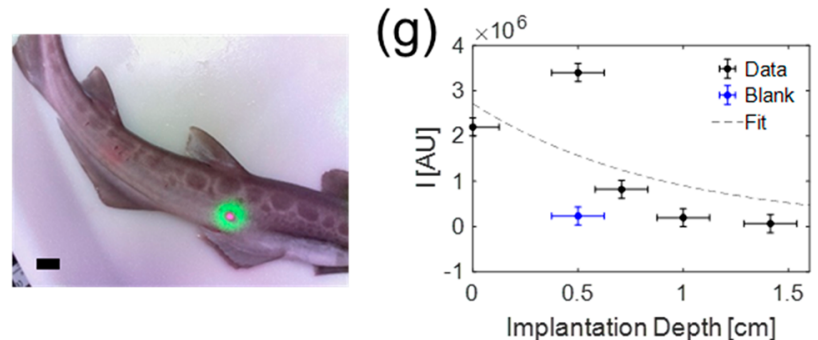

Figure 3. Effect of hydrogel implantation depth on fluorescence detection in teleosts (Sparus aurata and Stenotomus chrysops) and cat shark (Galeus melastomus). (a,b) Imaging setup and schematic. A fiber-coupled $561 \mathrm{~nm}$ laser fitted with an 850 short-pass filter was used to illuminate the implantation site before and after intramuscular delivery of hydrogels into previously deceased animals via trocar. The signal was collected by a Raspberry Pi camera connected to a 900 long-pass filter. The difference in gray values with and without the hydrogel was calculated. (c) In Sparus aurata, detectable hydrogel fluorescence decreased as injection depth was increased from just below the skin down to a limit of $0.7 \mathrm{~cm}$. A nonfluorescent hydrogel was injected just superficially below the skin and imaged to give the threshold difference in intensity for the signal to be attributable to the hydrogel and not to other artifacts, such as movement of the fish relative to the laser. (d) A superficially implanted SWNT-gel in Sparus aurata exhibited a steady fluorescence signal when imaged over $6 \mathrm{~min}$. (e) The detection limit of SWNT-gels in Stenotomus chrysops was $0.7 \mathrm{~cm}$. (f) Overlay of brightfield and fluorescence images of a fluorescent hydrogel implanted $0.5 \mathrm{~cm}$ below the skin in Galeus melastomus [scale $=20 \mathrm{~mm}]$. ( $\mathrm{g}$ ) SWNT-gels were detected down to a depth of $0.7 \mathrm{~cm}$ in Galeus melastomus, as compared to a nonfluorescent hydrogel implanted at a depth of $0.5 \mathrm{~cm}$.

(a)

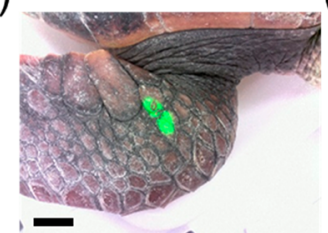

(d)

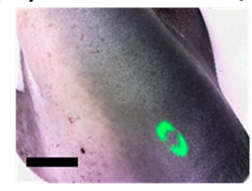

(b)

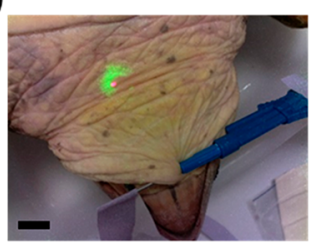

(f) (e)

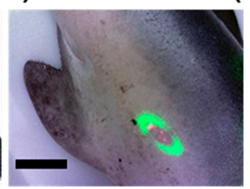

(c)

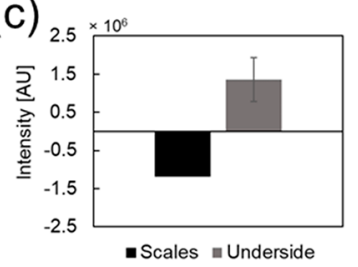

(g)

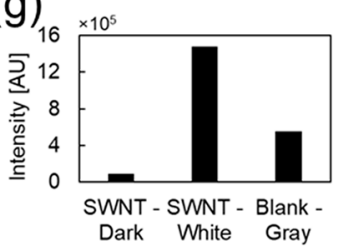

Figure 4. Detection of fluorescent hydrogels implanted superficially in optically heterogeneous tissues. Fluorescent hydrogels were implanted into (a) the scaly legs and (b) softer flesh beneath the neck of a sea turtle (Caretta caretta) (scale $=20 \mathrm{~mm}$ ). (c) Hydrogel fluorescence was detected in the neck but not the scaly legs. SWNT-gels were implanted subcutaneously in (d) dark and (e) white regions of a blue shark (Prionace glauca). A nonfluorescent hydrogel was implanted in (f) gray region of the tissue. ( $\mathrm{g}$ ) Fluorescence could be detected underneath white skin but not dark skin. The blank hydrogel in the gray region provided a baseline against which to determine fluorescence detection. Scale in all images is $20 \mathrm{~mm}$. 
260 autowhite balance gains, exposure times, and shutter speed were set 261 manually. The analog and digital gains were kept constant by 262 equilibration of the camera for a 1 min period.

263 Goldfish Hydrogel Implantations and Motion Tracking. All 264 experimental details below and associated husbandry procedures were 265 reviewed and approved by the Committee on Animal Care at MIT.

266 Two sarasa comet goldfish (Carassius auratus) were purchased from 267 LiveAquaria, housed in a $110 \mathrm{~L}$ glass aquarium with dimensions of $76 \times$ $26842 \times 30 \mathrm{~cm}^{3}$ (length $\times$ width $\times$ height), and allowed to acclimate for at 269 least 2 weeks prior to experimental manipulation. The water was 270 maintained at $24{ }^{\circ} \mathrm{C}$, and the aquarium was lit daily for $10 \mathrm{~h}$. Fish were 271 fed daily with flake foods (TetraFin).

272 Prior to implantation, hydrogels were treated under UV light for 15 $273 \mathrm{~min}$ and handled in a biological hood thereafter to ensure sterility. 274 Hydrogels were cut to a $1 \times 3 \times 1 \mathrm{~mm}^{3}$ shape and loaded into 16 gauge 275 needles. Fish were anesthetized in a solution of $60 \mathrm{mg} / \mathrm{L}$ tricaine 276 methanesulfonate. When the fish were nonresponsive to handling and a 277 fin pinch, the hydrogels were injected into muscle just below the dorsal 278 fin. The fish were allowed to recover in a holding tank before being 279 returned to the home tank.

280 To determine the impact of the hydrogel implant on the animal's 281 health, its movements were recorded using a surveillance system 282 consisting of the Raspberry Pi 2 computer with a Raspberry Pi Camera 283 Board v 2. Fish movements were extracted using the Kinovea software. 284 After the experimental lifetime, the fish were euthanized by 285 submersion into a $500 \mathrm{mg} / \mathrm{L}$ solution of tricaine methanesulfonate.

\section{RESULTS AND DISCUSSION}

287 Sensor Fabrication and in Vitro Optical Character288 ization. DNA-wrapped SWNT have been utilized in many 289 studies due to their high wrapping efficiency ${ }^{42,43}$ and flexibility 290 in selective sensing of different analytes. ${ }^{25-27,30,44}$ The UV-vis291 NIR absorption spectrum (Figure 2a) shows distinct peaks, 292 indicating successful nanoparticle suspension. Mass concen293 tration of total carbon in the solution was estimated using an 294 extinction coefficient of $\varepsilon_{632 \mathrm{~nm}}=0.036(\mathrm{mg} / \mathrm{L})^{-1} \mathrm{~cm}^{-1}$. ${ }^{45}$ Singly 295 dispersed ss $(\mathrm{AC})_{15}$-SWNT nanoparticles were produced at a 296 36\% yield based on a carbon mass balance.

297 Peak position and relative peak intensities of ss $(\mathrm{AC})_{15}$-SWNT 298 in solution phase or encapsulated in the hydrogel (SWNT-gel) 299 were identical in both the absorption spectra and fluorescence 300 emission spectra (Figure 2b), indicating that the dielectric 301 environments surrounding the SWNT were nearly identical. ${ }^{46,47}$ 302 The absorption spectrum of the SWNT-gel indicated a final 303 concentration of $33 \mathrm{mg} / \mathrm{L}$ SWNT. However, the fluorescence 304 intensity of the SWNT-gel was only $50 \%$ of the intensity in the 305 equivalent concentration in solution phase. Sample geometry 306 contributed to this decrease, as the hydrogels are only $1 \mathrm{~mm}$ in 307 thickness, whereas liquid samples were typically $1 \mathrm{~cm}$ in height. 308 Additionally, the chemical environment of the sensors in the 309 hydrogel is different, in that the SWNT are diffusionally 310 constrained by a polymer matrix. Free radicals that are generated 311 during the photopolymerization of the hydrogel may have also 312 chemically altered the DNA on the SWNT surface.

313 Characterization of SWNT-Gel Pore Size. The hydrogel 314 pore size formed by the spacing between cross-linked polymer 315 chains is a critical parameter that controls sensor functionality 316 and environment. The pores in the gel determine the size of the 317 analyte that is permitted to enter the network, as well as its rate 318 of diffusion, thereby affecting sensor response time. ${ }^{48}$ The pore 319 size can also be used to exclude large molecular weight 320 interfering molecules to improve sensor selectivity. Further321 more, the hydrogel's pore diameter relative to nanoparticle size 322 dictates the degree of nanoparticle entrapment. ${ }^{49}$ (a)

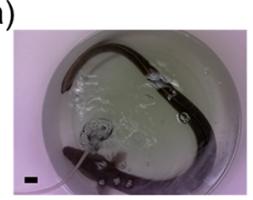

(d)

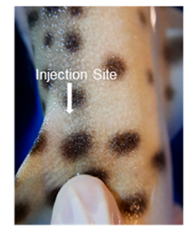

(g)

(e)
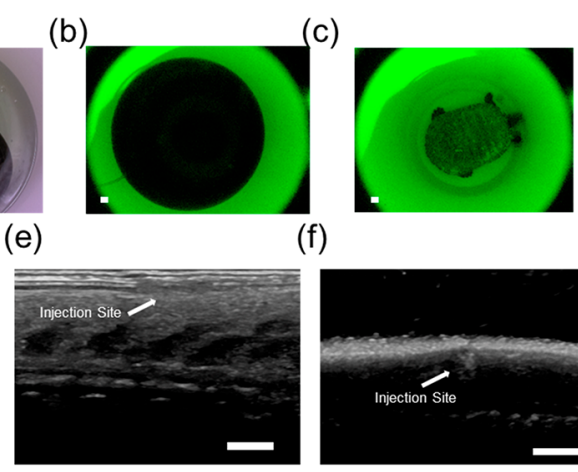

(f)

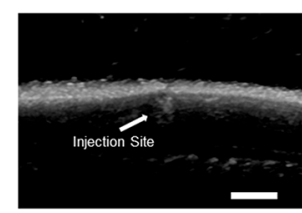

(h)

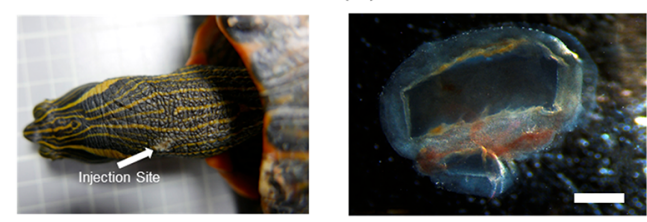

(i)

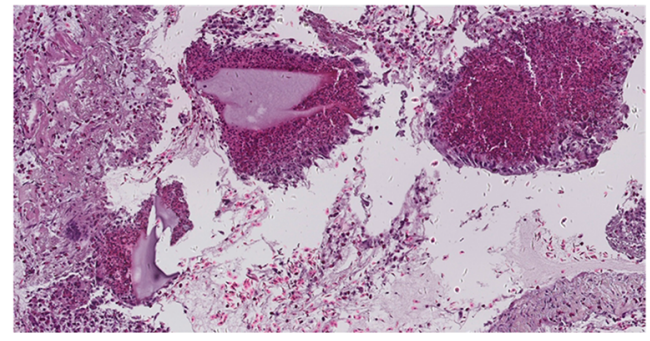

Figure 5. NIR fluorescent hydrogels implanted in a living European eel (Anguilla anguilla), eastern river cooter (Pseudemmys concinna), and catshark (Scyliorhinus stellaris). (a) Following implantation, attempts were made to track the fluorescence in the eel and turtle confined to a small space. (b,c) Dispersed laser excitation, animal movement, and long exposure times made these attempts unsuccessful in (b) the eel and (c) the turtle. All scalebars are $20 \mathrm{~mm}$. (d) The implantation site fully healed in the catshark by 33 days post-implantation. (e,f) High resolution ultrasound images were taken to examine noninvasively tissue response to the implant 4 weeks after implantation in the (e) eel and (f) catshark (scale $=5 \mathrm{~mm}$ ). The absence of significant changes in tissue architecture and echogenicity indicates that the hydrogels were well-tolerated in these organisms. (g) The injection site in the turtle did not heal completely 33 days post-implantation. (h) Hydrogels were removed from the turtle after 33 days and were found to be encapsulated by tissue. (i) Histology images from subcutaneous tissue surrounding the hydrogel implant in the turtle indicate a foreign body tissue reaction.

Swelling experiments were performed in $1 \times$ PBS to obtain the 323 average SWNT-gel pore size from the polymer network. The 324 swelling ratio was determined using the following equation 325

$$
Q=\frac{m_{\text {swollen }}}{m_{\text {dry }}}=\alpha^{-1}
$$

where $Q$ is the hydrogel swelling ratio and $m$ is the hydrogel 327 mass. $Q$ can then be used to calculate the average pore 328 diameter: ${ }^{50,51}$

$$
\begin{aligned}
& \bar{M}_{c}^{-1}=\frac{2}{\bar{M}_{n}}-\frac{\left(\bar{v} / V_{2}\right)\left[\ln (1-\alpha)+\alpha+\chi \alpha^{2}\right]}{\alpha^{1 / 3}-(2 / \theta) \alpha} \\
& \zeta=\alpha^{1 / 3}\left(\frac{2 C_{\infty} l^{2} \bar{M}_{c}}{M_{0}}\right)^{1 / 2}
\end{aligned}
$$


(a)

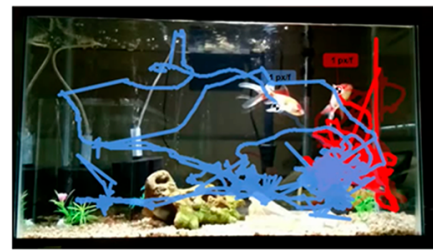

(b)

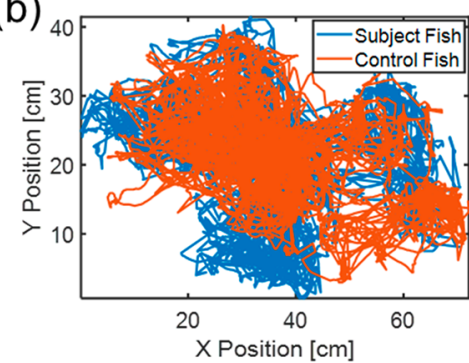

(c)

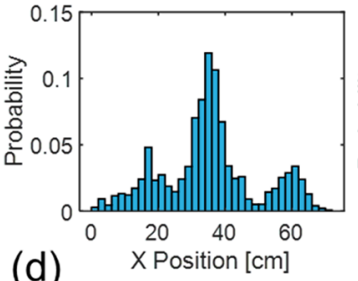

(d)

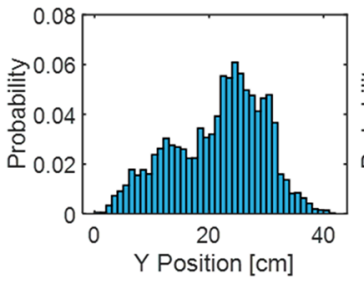

(e)
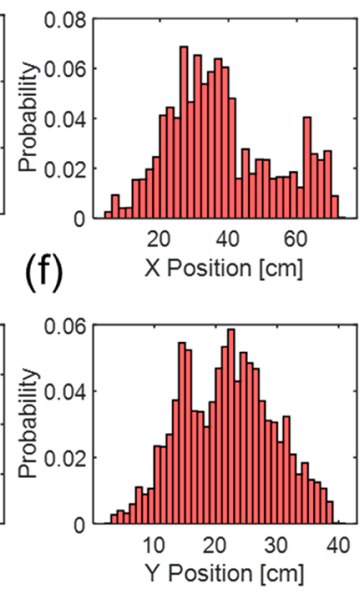

Figure 6. Quantification of hydrogel implant impact on animal health. (a) Snapshot of the capture video with corresponding sarasa comet goldfish ( Carassius auratus) movement trajectories included. Blue corresponds to a fish in which a nIR fluorescent hydrogel was implanted, while orange corresponds to a control fish without a hydrogel implant. (b) Trajectories of fish taken for $1 \mathrm{~h} 2$ days after a hydrogel was implanted into the subject fish. $(\mathrm{c}-\mathrm{f}) X$ and $Y$ position histograms for the subject fish $(\mathrm{c}, \mathrm{d})$ and control fish $(\mathrm{e}, \mathrm{f})$. The subject fish experienced neither impaired movement nor erratic movement due to the hydrogel implant, indicating good tolerance of the implant.

332 where $\bar{M}_{C}$ is the molecular weight between cross-links, $\bar{M}_{n}$ is the 333 molecular weight of the polymers without cross-linking $334(=8000), \bar{v}$ is the specific volume of the polymer $(=0.903$ $335 \mathrm{~mL} / \mathrm{g}), V_{2}$ is the specific volume of water $(=18.01 \mathrm{~mL} / \mathrm{mol}), \chi$ is 336 the Flory-Huggins parameter $(=0.3765), \theta$ is the functionality 337 of PEGDA $(=4), \xi$ is the average mesh side, $C_{\infty}$ is the Flory 338 characteristic ratio $(=6.9), l$ is the carbon-carbon bond length $339(=0.154 \mathrm{~nm})$, and $M_{0}$ is the molar mass of the repeat unit (= $34044.05 \mathrm{~g} / \mathrm{mol}$ ). The Flory parameter was obtained from a 341 previous study of PEG polymers. ${ }^{52}$ The average pore size was 342 estimated to be $15 \mathrm{~nm}$.

343 Raspberry Pi Imaging Systems. To understand the range 344 of organism-environment interactions and document variation 345 among individuals and populations, some biologging studies 346 have deployed sensors on anywhere from dozens to hundreds of 347 animals. ${ }^{7,53}$ To this end, some laboratory instruments are not 348 practical due to their prohibitive cost for large-scale deployment, 349 immobility, and fragility on a moving animal in its natural 350 environment. For example, InGaAs cameras typically used to 351 measure near-infrared fluorophores can weigh on the order of 5 $352 \mathrm{~kg}$ and can cost thousands of dollars. ${ }^{9,24,29}$ Consequently, we 353 chose to use inexpensive and portable Raspberry Pi computers 354 and cameras which cost on the order of tens of dollars. In the real 355 application, the components of a Raspberry Pi imaging system 356 can be readily incorporated into a miniaturized sensor suite. 357 Recently, Göröcs et al. incorporated a similar CMOS image 358 sensor into a portable imaging device weighing less than $40 \mathrm{~g} .{ }^{54}$ 359 Because the optical sensors on the cameras are fabricated from 360 silicon, which have limited sensitivity $(<0.1 \mathrm{~A} / \mathrm{W}$ above $980 \mathrm{~nm})$ 361 to the near-infrared fluorescence of SWNT (Figure S-1), we first 362 verified that the hydrogels could be visualized by our system 363 (Figure 2c,d). Analysis showed a linear trend of hydrogel 364 fluorescence with incident laser power density (Figure 2e).

365 Riboflavin as a Model Analyte for Chemical Sensing in 366 Vitro and ex Vivo. Riboflavin plays a key role in the recycling of $367 \mathrm{FADH}$ and $\mathrm{FAD}^{+}$in oxidative phosphorylation and is an 368 essential nutrient in a fish's diet. ${ }^{41,55}$ Riboflavin exists in plasma 369 typically between 1 and $100 \mathrm{nM}^{56,57}$ Furthermore, DNA oligonucleotides of various sequences, when complexed to 370 SWNTs, allow for a nIR fluorescence modulation in response to 371 riboflavin binding via both intensity quenching and wavelength 372 shifts, ${ }^{9}$ making it an ideal model analyte to evaluate in vivo 373 sensing feasibility.

374

The SWNT-gels showed stepwise decreases in fluorescence 375 with stepwise increases in surrounding riboflavin concentration, 376 with sensitivity from 1 to $100 \mu \mathrm{M}$ (Figure 2f). The calibration 377 curves were fitted to the following functional form:

378

$$
\text { Response }=\frac{I-I_{0}}{I_{0}}=\beta \frac{C}{C+K_{D}}
$$

where $\beta$ is the gain, $C$ is the riboflavin concentration, and $K_{\mathrm{D}}$ is 380 the equilibrium dissociation constant. To evaluate the perform- 381 ance of the Raspberry $\mathrm{Pi}$ relative to typical laboratory 382 equipment, we compared results obtained with a Princeton 383 Instrument 2D InGaAs camera. The calibration curves showed 384 good agreement (Figure $2 \mathrm{~g}$ ). For the InGaAs camera, $\beta$ was 385 -0.72 , and $K_{\mathrm{D}}$ was $11.3 \mu \mathrm{M}$, while the corresponding values 386 were -0.63 and $12.7 \mu \mathrm{M}$ for the Raspberry Pi. The difference in 387 maximum response is a product of higher background signal in 388 the Raspberry $\mathrm{Pi}$, which partially masked the fluorescence 389 quenching of the riboflavin. Future versions of the sensor tag will 390 be designed to eliminate such interference by optimizing optical 391 configurations and increase the sensitivity to detect physio- 392 logical levels of riboflavin.

393

Furthermore, the fluorescence of the SWNT-gels decreased in 394 response to a bolus of $100 \mu \mathrm{M}$ when placed in a 1-mm-thick skin 395 and muscle tissue sample of Stenotomus chrysops (Figure 2h). 396 The fluorescence decreased below the detection limit of the 397 Raspberry Pi camera.

398

Optical Penetration Depth. We constructed a simplified, 399 1-D mathematical model to describe the effects of material, 400 tissue, and equipment properties on the optical signal from a 401 sensor implanted into tissue. Incident excitation light is partially 402 reflected from the epidermal interface

$$
I_{0}=I_{i}\left(1-r_{\mathrm{ex}}\right)
$$


405 where $I_{i}$ and $I_{0}$ are the incident and transmitted excitation 406 fluences, respectively, and $r_{\mathrm{ex}}$ is the epidermal reflectivity at the 407 excitation wavelength. Tissue further attenuates excitation light 408 according to the Beer-Lambert law

$$
\log \left(\frac{I_{0}}{I}\right)=\gamma_{\text {ex }} d
$$

410 where $I$ is the fluence at the implantation site, $\gamma_{\mathrm{ex}}$ is the tissue 411 extinction coefficient at the excitation wavelength, and $d$ is 412 distance through tissue. The fluorescence intensity of the 413 hydrogel at the implantation site is described by

$$
\log \left(1-\frac{F_{0}}{\eta I A}\right)=-\epsilon_{\mathrm{ex}} c t
$$

415 where $F_{0}$ is the fluorescence intensity at the implantation site, $\eta$ 416 is the quantum efficiency of SWNT, $A$ is the cross-sectional area 417 of the hydrogel, $\varepsilon_{\mathrm{ex}}$ is the extinction coefficient of SWNT at the 418 excitation wavelength, $c$ is the concentration of SWNT in the 419 hydrogel, and $t$ is the hydrogel thickness. The thickness of the 420 hydrogel is assumed to be negligible compared to the 421 implantation depth. The fluorescence reaching the surface of 422 the epidermis is given by the following equation

$$
\log \left(\frac{F_{0}}{F}\right)=\gamma_{\mathrm{em}} d
$$

424 where $F$ is the fluorescence reaching the epidermal interface, $\gamma_{\mathrm{em}}$ 425 is the tissue extinction coefficient at the emission wavelength. 426 Back-reflection of fluorescence may occur at the epidermal 427 interface

$428 \quad F_{\mathrm{f}}=F\left(1-r_{\mathrm{em}}\right)$

429 where $F_{\mathrm{f}}$ is the fluorescence exiting the tissue and $r_{\mathrm{em}}$ is the 430 reflectivity at the fluorescent wavelength. Assuming minimal 431 scattering and absorption between the epidermal surface and the 432 photodetector, the measured signal is described by

$433 \quad S=F_{\mathrm{f}} R$

434 where $S$ is the signal, and $R$ is the responsivity of the camera. 435 Combining eqs $5-10$ yields

$$
\begin{aligned}
& \log \left(\frac{S}{\eta A R I_{i}\left(1-r_{\mathrm{ex}}\right)\left(1-r_{\mathrm{em}}\right)}\right) \\
& \quad=\log \left(1-10^{\varepsilon_{\mathrm{ex}} c t}\right)-d\left(\gamma_{\mathrm{ex}}+\gamma_{\mathrm{em}}\right)
\end{aligned}
$$

437 The terms in eq 11 can be classified into material, tissue, and 438 equipment properties and tunable engineering parameters. The 439 specific fluorophore dictates the value of $\eta$ and $\varepsilon_{\mathrm{ex}}$. Different 440 tissues attenuate light transmission to varying extents and 441 consequently have unique values of $\gamma$, which may be measured in 442 a future study via light transmission measurements. Both 443 absorption and scattering contribute to the extinction 444 coefficient. Scattering decreases with increasing incident 445 wavelength, ${ }^{58}$ while absorption is largely determined by water 446 and blood absorption, which is minimal in the SWNT 447 fluorescent region. ${ }^{59}$ Furthermore, unlike organic fluorophores, 448 SWNT do not photobleach and thus exhibit a constant $c$ as long 449 as the implant maintains its integrity. Thus, the near-infrared 450 fluorescence of SWNT is ideal for an in vivo optical biosensor 451 due to the lack of photobleaching and the transparency of the 452 near-infrared window. ${ }^{60,61}$ Controllable parameters include $I_{i}, A$, $453 c, t, r$, and $d$. Increased hydrogel thickness, fluorophore concentration, equipment responsivity, and excitation power 454 and decreased implantation depth increase the fluorescence 455 signal. In a previous study, Iverson et al. measured the 456 fluorescence of an alginate hydrogel with $10 \mathrm{mg} / \mathrm{L}$ SWNT to 457 a depth of $5 \mathrm{~mm}$ in tissue phantoms using a hyperspectral CRI 458 Maestro system. ${ }^{62}$

For this application, consideration of the marine organism 460 tissue properties is critical. Many fish species, including teleosts, 461 have evolved skin containing significant amounts of reflective 462 guanine crystals in the stratum argenteum and underneath the 463 scales, which may camouflage the animal against predators. ${ }^{63}{ }_{464}$ The reflective spectra of such biomaterials have been thoroughly 465 characterized in previous work. ${ }^{64}$ These different skin types will 466 affect the penetration of light through tissue. Others, such as 467 sharks and marine reptiles, have evolved thick, mechanically stiff 468 skin and/or scales as protection against environmental hazards, 469 which may require specialized methods of placing implantable 470 devices. $^{65}$ All together, these factors suggest that each species 471 should be considered individually when using implantable nIR 472 fluorescent hydrogel sensors for biologging. 473

Two deceased teleosts (Sparus aurata and Stenotomus 474 chrysops), a female adult catshark (Galeus melastormus) were 475 used for the nIR penetration versus depth study. The teleosts 476 were chosen because over 32,500 species exist, making them the 477 largest category of vertebrates. ${ }^{66}$ Furthermore, catsharks 478 comprise over $10 \%$ of extant cartilaginous fish. ${ }^{67}$ Images were 479 taken of the fish before and after placement of the hydrogel using 480 the Raspbery Pi camera system (Figure 3a,b). Movement of the 481 animal relative to the imaging setup was minimized such that 482 differences in signal between the two images is predominantly 483 the hydrogel, not position change. In Sparus aurata, the nIR 484 fluorescent SWNT-gels were detected up to a depth of $7 \mathrm{~mm} 485$ (Figure 3c). Injection of sham nonfluorescent hydrogels using 486 the same method verified that the difference in signal due to 487 movement was negligible compared to the additional signal from 488 the fluorescence. The residual signal is a small change in laser 489 reflection from a small shift in position of the fish tissue. The 490 SWNT-gels also exhibited stable fluorescence (Figure 3d). This 491 stability is critical, so that perturbations can be attributed solely 492 to changes in analyte concentrations. For Stenotomus chrysops 493 and Galeus melastormus (Figure $3 \mathrm{e}-\mathrm{g}$ ), the SWNT-gels were 494 detected again to a depth of $7 \mathrm{~mm}$ over a minimum signal 495 difference threshold determined by injection of a nonfluorescent 496 hydrogel (Figure 3g). A simplified version of eq 11 was used to 497 fit the data and reproduced the trends.

$$
S=a \cdot 10^{b \cdot d+c}
$$

The fit parameters are reported in Supporting Information. $\quad 500$

As can be seen in Figure 3c,e,g, there was not a monotonic 501 decrease in fluorescence with increasing depth. We attribute the 502 noise to variations in hydrogel thickness, cross-sectional area, 503 placement at the intended depth, and position relative to the 504 excitation source. As illustrated by eq 11 , variations in geometry 505 and placement of the SWNT-gels necessarily change the signal 506 by reducing the excitation power incident on the hydrogel and 507 changing the attenuation distance of the excitation and 508 fluorescence through tissue.

509

Although the penetration depth of the SWNT sensors in the 510 target species were similar to that of previous studies, ${ }^{62}$ the 511 maximum depth can be increased using several approaches. 512 First, the excitation and fluorescence detection equipment can 513 be optimized specifically for SWNT-based biosensors. An 514 InGaAs photodetector, which has almost an order of magnitude 515 
516 higher photoresponsivity $(0.67 \mathrm{~A} / \mathrm{W}$ at $1000 \mathrm{~nm})$ may replace 517 the silicon-based camera $(0.067 \mathrm{~A} / \mathrm{W}$ at $1000 \mathrm{~nm})$ used in this 518 study (Figure S-1). This equipment, along with other optical 519 components such as lenses, can be attached directly to the 520 animal instead of being placed at standoff distances, thus 521 reducing the optical path length, optimizing excitation and 522 fluorescence collection, and increasing signal. Alternatively, 523 optical fibers may be implanted transdermally, in the form of an 524 optode, to couple the excitation source directly with the 525 hydrogel and the hydrogel with the photodetector. ${ }^{68}$

526 Ultimately, placement of the sensor could be influenced by 527 other factors in addition to optical penetration depth, including 528 local analyte concentration and sensor sensitivity. Many analytes 529 of interest, such as glucose, cortisol, and vitamins, exist in 530 interstitial fluids and can be theoretically queried with a hydrogel 531 implanted superficially atop the hypodermis. ${ }^{69}$

532 Tissue Heterogeneity. Different color patterns of tissue 533 and mechanically distinct exteriors may exist on the skin of the 534 same animal, which may affect hydrogel implantation and/or 535 fluorescence visibility. To examine this issue, we implanted 536 hydrogels in different skin tissues of a juvenile female sea turtle ( 537 Caretta caretta) and a juvenile male blue shark (Prionace glauca). 538 The sea turtle had both scaly and fleshy regions of the skin, ${ }^{65}$ 539 whereas the blue shark had distinctly colored regions ranging 540 from dark blue to white. ${ }^{70}$

541 SWNT-gels were delivered in both the front right leg and the 542 flesh centered underneath the neck of the sea turtle (Figure $5434 \mathrm{a}, \mathrm{b})$. As the needle could not pierce the scales, it was inserted 544 between them. The neck flesh was stretched prior to hydrogel 545 placement to prevent folding of additional skin on top of the 546 implant, avoiding artificial increases in the optical path length. 547 The hydrogel was not visible beneath the scales but was visible 548 beneath the fleshy skin of the neck (Figure 4c). SWNT-gel 549 sensors were placed underneath the white and dark sections of 550 shark's epidermis, and a nonfluorescent hydrogel was placed into 551 a gray area to provide a baseline against which nIR fluorescent 552 hydrogels could be compared (Figure 4d-f). The nIR 553 fluorescent hydrogel was visible beneath the white but not the 554 dark-colored epidermis (Figure 4g).

555 In both organisms, dark sections of tissue masked the nIR 556 fluorescence of the sensor implants. Increased melanin levels in 557 the epidermis result in higher absorption coefficients up to 1100 $558 \mathrm{~nm},{ }^{71,72}$ resulting in less excitation of the hydrogel and 559 transmission of $(6,5)$ SWNT fluorescence by increasing the 560 values of $\gamma_{\mathrm{ex}}$ and $\gamma_{\mathrm{em}}$ in eq 11 . UV-vis-NIR absorption 561 measurements of tissue samples can quantify these wave562 length/tissue dependent effects in a future study.

563 These results indicate two additional requirements for nIR 564 fluorescent biosensors for in vivo applications. First, to maximize 565 the signal-to-noise ratio, sensors should be delivered to tissues 566 that are as optically transparent as possible for both the 567 excitation and emission wavelengths. Furthermore, sensor 568 fluorescence may have to be normalized against an invariant 569 internal standard to eliminate the effects of tissue hetero570 geneity. ${ }^{44}$ Second, to deliver hydrogels via a minimally invasive 571 injection, some tissue sections will be inaccessible due to their 572 mechanical strength and rigidity.

573 Imaging and Sensor Operation in Live Animals. Several 574 questions regarding tolerance/biocompatibility of the implant 575 and its effects on behavior can only be answered using living 576 animals. A moving animal also adds greater complexity when 577 imaging which may require reconfigurations of the sensor.
A female adult European eel (Anguilla anguilla), a female adult 578 eastern river cooter (Pseudemmys concinna), and a juvenile male 579 catshark (Scyliorhinus stellaris) were tagged with sensor 580 hydrogels and monitored for up to 2 months. We attempted 581 to image the eel and turtle in a small bucket from a distance of 582 $0.5 \mathrm{~m}$, but were unsuccessful for several reasons (Figure $5 \mathrm{a}-\mathrm{c}$ ). 583 First, the camera and excitation sources were moved farther 584 away to image the entire field of view. This reduced both the 585 excitation power density incident upon the surface of the 586 epidermis from 150 to $0.3 \mathrm{~mW} / \mathrm{cm}^{2}$ and consequently the 587 fluorescence upon the camera's sensor by a factor of at least 500, 588 according to eq 11. Furthermore, the combination of a long 589 exposure time and animal movement apparently blurred the 590 images.

591

Engineering Design for nIR Fluorescent Hydrogel 592 Implants. A central goal of the current work is to utilize these 593 findings to design sensing hydrogel implants. A wearable 594 fluorescence reader that conforms to the animal's body as it 595 moves is necessary. ${ }^{73}$ Fixing the position of the measurement 596 unit relative to the SWNT-gels eliminates changes in hydrogel 597 fluorescence due to a changing excitation field and/or 598 misalignment of the hydrogel and camera. Furthermore, placing 599 the measurement device directly on top of the hydrogel reduces 600 the optical path length, increasing the signal-to-noise ratio. As 601 such, the miniaturization into and attachment methods of a 602 flexible form factor are critical next steps.

603

Biocompatibility of the hydrogel was favorable in two of the 604 three animals. We found no changes in movement or feeding 605 behavior of the eel and catshark for two months post- 606 implantation (Figure 5d). In the ultrasound images, the 607 implantation site was identified via a slight change in tissue 608 structure and echogenicity, but the surrounding tissue was 609 completely normal (Figure 5e,f). In the case of a significant 610 foreign body reaction, larger changes in architecture and 611 echogenicity would be found in the periphery of the implant 612 as it becomes encapsulated. ${ }^{74,75}$ In contrast, histopathology 613 suggested that the turtle experienced some reaction to the 614 implant. The injection site did not heal cleanly (Figure 5g). It is 615 important to note that there may have been an infection of the 616 wound following implantation, precluding clean healing. 617 Granules containing hydrogel fragments were extracted from 618 the implantation site one month after the procedure (Figure 5h). 619 $H \& E$ stained tissue sections showed infiltration of inflammatory 620 cells into the deep dermis, hypodermis, and cutaneous muscle. 621 The infiltrate consisted of heterophiles, macrophages, and 622 several multinucleated giant cells, consistent with panniculitis 623 and a foreign body reaction to the implant (Figure 5i). However, 624 no behavioral changes were noted in the turtle.

625

A similar implantation procedure was performed on adult 626 Sarasa comet goldfish (Carassius auratus), and its movement 627 patterns were analyzed relative to a control goldfish without an 628 implant (Figure 6a). Animal trajectories and position histograms 629 did not differ significantly between the two animals, indicating 630 that the hydrogel implants do not adversely impact animal 631 health (Figure $6 \mathrm{~b}-\mathrm{f}$ ). During times of stress or infections, the 632 fish may swim violently or erratically. In the case of serious 633 illness, fish movement would slow severely. ${ }^{76,77}$ The video data 634 and position histograms (Figure 6c-f) show that the subject fish 635 showed neither erratic movement nor stationary behavior 636 relative to the control. The absence of other abnormalities, 637 such as damaged fins, disinterest in food, and discoloration, 638 further indicate that the fish tolerated the implant well. ${ }^{78} 639$ 
640 Furthermore, goldfish were maintained up to six months with 641 the hydrogel implant, indicating long-term biocompatibility. 642 These results effectively form a pilot study that can be used to 643 direct and prioritize future work involving larger sample sizes, a 644 greater diversity of species, optimization of the hydrogel and 645 delivery method, and development of a wearable fluorescence 646 reader.

\section{CONCLUSIONS}

648 In summary, the feasibility of applying CoPhMoRe sensors for 649 the physiological biologging of marine organisms was 650 demonstrated in nine species of aquatic vertebrates. Future 651 work will perform similar tissue penetration, tissue hetero652 geneity, and biocompatibility studies with a larger number of 653 animals to probe phenotypic diversity. Strategies to normalize 654 sensor signals against individual implant site optical properties 655 and internal fluorescent standards will be explored to create 656 absolute interspecies calibrations. Ratiometric approaches to 657 optical sensing will mitigate movement and other artifacts that 658 may confound the signal. ${ }^{44}$ The successful measurement of the 659 fluorescent hydrogels using an inexpensive, field portable 660 Raspberry Pi imaging setup motivates further efforts to design 661 a wearable, flexible sensor tag that integrates optoelectronic 662 components tailored for physiological biologging using SWNT663 gels. These technical improvements may improve the signal-to664 noise ratio, time resolution of the measurements, and stability of 665 the signal when attached to a moving animal. In parallel, the 666 underlying SWNT nanosensors may be engineered to be 667 sensitive to a wider range of bioanalytes to investigate a wider 668 range of physiological states. The detection range of the 669 riboflavin sensor described herein will be further improved to be 670 sensitive to the physiologically relevant range. This work 671 advances the application of biosensors into animals beyond 672 the commonly used rodent and zebrafish models and carves a 673 path toward the physiological biologging of aquatic organisms.

\section{ASSOCIATED CONTENT}

\section{S Supporting Information}

676 The Supporting Information is available free of charge on the 677 ACS Publications website at DOI: 10.1021/acssen678 sors. 8 b00538.

679 Fit parameters for optical penetration depth model, 680 comparison of SWNT fluorescence and photoresponsiv681 ity of common photodetectors (PDF)

\section{$682 \square$ AUTHOR INFORMATION}

683 Corresponding Author

684 *E-mail: strano@mit.edu.

685 ORCID

686 Michael S. Strano: 0000-0003-2944-808X

687 Notes

688 The authors declare no competing financial interest.

\section{ACKNOWLEDGMENTS}

690 This research was supported by the King Abdullah University of 691 Science \& Technology (OSR-2015 Sensors 2707). FTN is 692 supported by the Arnold and Mabel Beckman Foundation 693 through the Arnold O. Beckman Postdoctoral Fellowship. The 694 authors are also grateful to the Oceanogràic animal care staff for 695 performing histological analysis, helpful discussions, and 696 financial support (OCE-10-18) in performing this research.
We also thank the Health and Animal Pathology Group, Dpt. 697 PASAPTA, Facultad de Veterinaria, Universidad Cardenal 698 Herrera-CEU, CEU Universities, for performing histological 699 analysis and interpretation.

700

\section{ABBREVIATIONS}

701

SWNT, single wall carbon nanotube; CoPhMoRe, corona phase 702 molecular recognition; nIR, near-infrared; PEGDA, poly- 703 (ethylene glycol) diacrylate; ss $(\mathrm{AC})_{15}$, single-stranded (AC) $)_{15} 704$ DNA; InGaAs, Indium gallium arsenide

705

\section{REFERENCES}

706

(1) McIntyre, T. Trends in Tagging of Marine Mammals: A Review of 707 Marine Mammal Biologging Studies. African J. Mar. Sci. 2014, 36 (4), 708 409-422.

709

(2) Cooke, S. J.; Brownscombe, J. W.; Raby, G. D.; Broell, F.; Hinch, S. 710 G.; Clark, T. D.; Semmens, J. M. Remote Bioenergetics Measurements 711 in Wild Fish: Opportunities and Challenges. Comp. Biochem. Physiol., 712 Part A: Mol. Integr. Physiol. 2016, 202, 23-37.

713

(3) Watanabe, Y. Y.; Ito, M.; Takahashi, A. Testing Optimal Foraging 714 Theory in a Penguin-krill System. Proc. R. Soc. London, Ser. B 2014, 281715 (1779), 20132376.

716

(4) Amélineau, F.; Fort, J.; Mathewson, P. D.; Speirs, D. C.; Courbin, 717 N.; Perret, S.; Porter, W. P.; Wilson, R. J.; Grémillet, D. Energyscapes 718 and Prey Fields Shape a North Atlantic Seabird Wintering Hotspot 719 under Climate Change. R. Soc. Open Sci. 2018, 5, 171883.

(5) Barnett, A.; Payne, N. L.; Semmens, J. M.; Fitzpatrick, R. 721 Ecotourism Increases the Field Metabolic Rate of Whitetip Reef Sharks. 722 Biol. Conserv. 2016, 199, 132-136.

723

(6) Baylis, A. M.M.; Orben, R. A.; Arnould, J. P.Y.; Peters, K.; Knox, 724 T.; Costa, D. P.; Staniland, I. J. Diving Deeper into Individual Foraging 725 Specializations of a Large Marine Predator, the Southern Sea Lion. 726 Oecologia 2015, 179 (4), 1053-1065.

727

(7) Hays, G. C.; Scott, R. Global Patterns for Upper Ceilings on 728 Migration Distance in Sea Turtles and Comparisons with Fish, Birds 729 and Mammals. Funct. Ecol. 2013, 27 (3), 748-756. 730

(8) Yasuda, T.; Kawabe, R.; Takahashi, T.; Murata, H.; Kurita, Y.; 731 Nakatsuka, N.; Arai, N. Habitat Shifts in Relation to the Reproduction 732 of Japanese Flounder Paralichthys Olivaceus Revealed by a Depth- 733 Temperature Data Logger. J. Exp. Mar. Biol. Ecol. 2010, 385 (1-2), 50- 734 58.

(9) Zhang, J.; Landry, M. P.; Barone, P. W.; Kim, J.-H.; Lin, S.; Ulissi, 736 Z. W.; Lin, D.; Mu, B.; Boghossian, A. A.; Hilmer, A. J.; et al. Molecular 737 Recognition Using Corona Phase Complexes Made of Synthetic 738 Polymers Adsorbed on Carbon Nanotubes. Nat. Nanotechnol. 2013, 8739 (12), 959-968.

740

(10) Wu, D.; Sedgwick, A. C.; Gunnlaugsson, T.; Akkaya, E. U.; Yoon, 741 J.; James, T. D. Fluorescent Chemosensors: The Past, Present and 742 Future. Chem. Soc. Rev. 2017, 46 (23), 7105-7123.

743

(11) Xiao, T.; Wu, F.; Hao, J.; Zhang, M.; Yu, P.; Mao, L. In Vivo 744 Analysis with Electrochemical Sensors and Biosensors. Anal. Chem. 745 2017, 89 (1), 300-313.

\section{6}

(12) Sun, W.; Guo, S.; Hu, C.; Fan, J.; Peng, X. Recent Development 747 of Chemosensors Based on Cyanine Platforms. Chem. Rev. 2016, 116748 (14), 7768-7817.

(13) Soto, R. J.; Hall, J. R.; Brown, M. D.; Taylor, J. B.; Schoenfisch, M. 750 H. In Vivo Chemical Sensors: Role of Biocompatibility on Performance 751 and Utility. Anal. Chem. 2017, 89 (1), 276-299. 752

(14) Rong, G.; Corrie, S. R.; Clark, H. A. In Vivo Biosensing: Progress 753 and Perspectives. ACS Sensors 2017, 2 (3), 327-338. 754

(15) Howes, P. D.; Chandrawati, R.; Stevens, M. M. Colloidal 755 Nanoparticles as Advanced Biological Sensors. Science (Washington, 756 DC, U. S.) 2014, 346 (6205), 1247390.

757

(16) Carter, K. P.; Young, A. M.; Palmer, A. E. Fluorescent Sensors for 758 Measuring Metal Ions in Living Systems. Chem. Rev. 2014, 114 (8), 759 4564-4601. 
761 (17) Scholten, K.; Meng, E. A Review of Implantable Biosensors for 762 Closed-Loop Glucose Control and Other Drug Delivery Applications. 763 Int. J. Pharm. 2018, 544 (2), 319-334.

764 (18) Cotruvo, J. A., Jr.; Aron, A. T.; Ramos-Torres, K. M.; Chang, C. J. 765 Synthetic Fluorescent Probes for Studying Copper in Biological 766 Systems. Chem. Soc. Rev. 2015, 44 (13), 4400-4414.

767 (19) Sun, K.; Yang, Y.; Zhou, H.; Yin, S.; Qin, W.; Yu, J.; Chiu, D. T.; 768 Yuan, Z.; Zhang, X.; Wu, C. Ultrabright Polymer-Dot Transducer 769 Enabled Wireless Glucose Monitoring via a Smartphone. ACS Nano 770 2018, 12 (6), 5176-5184.

771 (20) Deng, B.; Ren, M.; Kong, X.; Zhou, K.; Lin, W. Development of 772 an Enhanced Turn-on Fluorescent $\mathrm{HOCl}$ Probe with a Large Stokes 773 Shift and Its Use for Imaging $\mathrm{HOCl}$ in Cells and Zebrafish. Sens. 774 Actuators, B 2018, 255, 963-969.

775 (21) Gurkov, A.; Sadovoy, A.; Shchapova, E.; Teh, C.; Meglinski, I.; 776 Timofeyev, M. Microencapsulated Fluorescent PH Probe as Implant777 able Sensor for Monitoring the Physiological State of Fish Embryos. 778 PLoS One 2017, 12 (10), e0186548.

779 (22) Ferreira, N. R.; Ledo, A.; Laranjinha, J.; Gerhardt, G. A.; Barbosa, 780 R. M. Simultaneous Measurements of Ascorbate and Glutamate in Vivo 781 in the Rat Brain Using Carbon Fiber Nanocomposite Sensors and 782 Microbiosensor Arrays. Bioelectrochemistry 2018, 121, 142-150.

783 (23) Mehrotra, P. Biosensors and Their Applications - A Review. J. 784 Oral Biol. Craniofacial Res. 2016, 6 (2), 153-159.

785 (24) Bisker, G.; Dong, J.; Park, H. D.; Iverson, N. M.; Ahn, J.; Nelson, 786 J. T.; Landry, M. P.; Kruss, S.; Strano, M. S. Protein-Targeted Corona 787 Phase Molecular Recognition. Nat. Commun. 2016, 7, 10241.

788 (25) Zhang, J. Q.; Boghossian, A. A.; Barone, P. W.; Rwei, A.; Kim, J. 789 H.; Lin, D. H.; Heller, D. A.; Hilmer, A. J.; Nair, N.; Reuel, N. F.; et al. 790 Single Molecule Detection of Nitric Oxide Enabled by d(AT)(15) 791 DNA Adsorbed to Near Infrared Fluorescent Single-Walled Carbon 792 Nanotubes. J. Am. Chem. Soc. 2011, 133 (3), 567-581.

793 (26) Jin, H.; Heller, D. a; Kalbacova, M.; Kim, J.-H.; Zhang, J.; 794 Boghossian, A. a; Maheshri, N.; Strano, M. S. Detection of Single795 Molecule H2O2 Signalling from Epidermal Growth Factor Receptor 796 Using Fluorescent Single-Walled Carbon Nanotubes. Nat. Nanotechnol. 797 2010, 5 (4), 302-309.

798 (27) Kruss, S.; Landry, M. P.; Vander Ende, E.; Lima, B. M.A.; Reuel, 799 N. F.; Zhang, J.; Nelson, J.; Mu, B.; Hilmer, A.; Strano, M. 800 Neurotransmitter Detection Using Corona Phase Molecular Recog801 nition on Fluorescent Single-Walled Carbon Nanotube Sensors. J. Am. 802 Chem. Soc. 2014, 136 (2), 713-724.

803 (28) Kruss, S.; Salem, D. P.; Vuković, L.; Lima, B.; Vander Ende, E.; 804 Boyden, E. S.; Strano, M. S. High-Resolution Imaging of Cellular 805 Dopamine Efflux Using a Fluorescent Nanosensor Array. Proc. Natl. 806 Acad. Sci. U. S. A. 2017, 114 (8), 1789-1794.

807 (29) Bisker, G.; Bakh, N. A.; Lee, M. A.; Ahn, J.; Park, M.; O'Connell, 808 E. B.; Iverson, N. M.; Strano, M. S. Insulin Detection Using a Corona 809 Phase Molecular Recognition Site on Single-Walled Carbon Nano810 tubes. ACS Sensors 2018, 3 (2), 367-377.

811 (30) Iverson, N. M.; Barone, P. W.; Shandell, M.; Trudel, L. J.; Sen, S.; 812 Sen, F.; Ivanov, V.; Atolia, E.; Farias, E.; McNicholas, T. P.; et al. In Vivo 813 Biosensing via Tissue-Localizable near-Infrared-Fluorescent Single814 Walled Carbon Nanotubes. Nat. Nanotechnol. 2013, 8 (11), 873-880. 815 (31) Hays, G. C.; Ferreira, L. C.; Sequeira, A. M.M.; Meekan, M. G.; 816 Duarte, C. M.; Bailey, H.; Bailleul, F.; Bowen, W. D.; Caley, M. J.; 817 Costa, D. P.; et al. Key Questions in Marine Megafauna Movement 818 Ecology. Trends Ecol. Evol. 2016, 31 (6), 463-475.

819 (32) Payne, N. L.; Taylor, M. D.; Watanabe, Y. Y.; Semmens, J. M. 820 From Physiology to Physics: Are We Recognizing the Flexibility of 821 Biologging Tools? J. Exp. Biol. 2014, 217 (3), 317-322.

822 (33) Wilson, R. P.; Gómez-Laich, A.; Sala, J. E.; Dell’Omo, G.; Holton, 823 M. D.; Quintana, F. Long Necks Enhance and Constrain Foraging 824 Capacity in Aquatic Vertebrates. Proc. R. Soc. London, Ser. B 2017, 284 825 (1867), 20172072.

826 (34) Meekan, M. G.; Fuiman, L. A.; Davis, R.; Berger, Y.; Thums, M. 827 Swimming Strategy and Body Plan of the World's Largest Fish: 828 Implications for Foraging Efficiency and Thermoregulation. Front. Mar. 829 Sci. 2015, 2, 64.
(35) Lee, M. A.; Bakh, N.; Bisker, G.; Brown, E. N.; Strano, M. S. A 830 Pharmacokinetic Model of a Tissue Implantable Cortisol Sensor. Adv. 831 Healthcare Mater. 2016, 5 (23), 3004-3015.

832

(36) Pon, L. B.; Hinch, S. G.; Cooke, S. J.; Patterson, D. A.; Farrell, A. 833 P. Physiological, Energetic and Behavioural Correlates of Successful 834 Fishway Passage of Adult Sockeye Salmon Oncorhynchus Nerka in the 835 Seton River, British Columbia. J. Fish Biol. 2009, 74 (6), 1323-1336. 836

(37) Crossin, G. T.; Takahashi, A.; Sakamoto, K. Q.; Trathan, P. N.; 837 Williams, T. D. Habitat Selection by Foraging Macaroni Penguins 838 Correlates with Hematocrit, an Index of Aerobic Condition. Mar. Ecol.: 839 Prog. Ser. 2015, 530, 163-176.

840

(38) O’Toole, A. C.; Dechraoui Bottein, M. Y.; Danylchuk, A. J.; 841 Ramsdell, J. S.; Cooke, S. J. Linking Ciguatera Poisoning to Spatial 842 Ecology of Fish: A Novel Approach to Examining the Distribution of 843 Biotoxin Levels in the Great Barracuda by Combining Non-Lethal 844 Blood Sampling and Biotelemetry. Sci. Total Environ. 2012, 427-428, 845 98-105.

846

(39) Kruss, S.; Hilmer, A. J.; Zhang, J.; Reuel, N. F.; Mu, B.; Strano, M. 847 S. Carbon Nanotubes as Optical Biomedical Sensors. Adv. Drug Delivery 848 Rev. 2013, 65 (15), 1933-1950.

849

(40) Bisker, G.; Iverson, N. M.; Ahn, J.; Strano, M. S. A 850 Pharmacokinetic Model of a Tissue Implantable Insulin Sensor. Adv. 851 Healthcare Mater. 2015, 4 (1), 87-97.

852

(41) Massey, V. The Chemical and Biological Versatility of Riboflavin. 853 Biochem. Soc. Trans. 2000, 28 (4), 283-296.

854

(42) Nakashima, N.; Okuzono, S.; Murakami, H.; Nakai, T.; 855 Yoshikawa, K. DNA Dissolves Single-Walled Carbon Nanotubes in 856 Water. Chem. Lett. 2003, 32 (5), 456-457. 857

(43) Zheng, M.; Jagota, A.; Semke, E. D.; Diner, B. A.; McLean, R. S.; 858 Lustig, S. R.; Richardson, R. E.; Tassi, N. G. DNA-Assisted Dispersion 859 and Separation of Carbon Nanotubes. Nat. Mater. 2003, 2 (5), 338- 860 342.

861

(44) Giraldo, J. P.; Landry, M. P.; Kwak, S. Y.; Jain, R. M.; Wong, M. 862 H.; Iverson, N. M.; Ben-Naim, M.; Strano, M. S. A Ratiometric Sensor 863 Using Single Chirality Near-Infrared Fluorescent Carbon Nanotubes: 864 Application to in Vivo Monitoring. Small 2015, 11 (32), 3973-3984. 865

(45) Zhang, J.; Kruss, S.; Hilmer, A. J.; Shimizu, S.; Schmois, Z.; De La 866 Cruz, F.; Barone, P. W.; Reuel, N. F.; Heller, D. A.; Strano, M. S. A 867 Rapid, Direct, Quantitative, and Label-Free Detector of Cardiac 868 Biomarker Troponin T Using Near-Infrared Fluorescent Single-Walled 869 Carbon Nanotube Sensors. Adv. Healthcare Mater. 2014, 3 (3), 412- 870 423.

871

(46) Larsen, B. A.; Deria, P.; Holt, J. M.; Stanton, I. N.; Heben, M. J.; 872 Therien, M. J.; Blackburn, J. L. Effect of Solvent Polarity and 873 Electrophilicity on Quantum Yields and Solvatochromic Shifts of 874 Single-Walled Carbon Nanotube Photoluminescence. J. Am. Chem. Soc. 875 2012, 134 (30), 12485-12491.

(47) Choi, J. H.; Strano, M. S. Solvatochromism in Single-Walled 877 Carbon Nanotubes. Appl. Phys. Lett. 2007, 90 (22), 88-91. 878

(48) Tokarev, I.; Minko, S. Stimuli-Responsive Porous Hydrogels at 879 Interfaces for Molecular Filtration, Separation, Controlled Release, and 880 Gating in Capsules and Membranes. Adv. Mater. 2010, 22 (31), 3446- 881 3462.

(49) Zhao, F.; Yao, D.; Guo, R.; Deng, L.; Dong, A.; Zhang, J. 883 Composites of Polymer Hydrogels and Nanoparticulate Systems for 884 Biomedical and Pharmaceutical Applications. Nanomaterials 2015, 5885 (4), 2054-2130.

(50) Peppas, N. A.; Huang, Y.; Torres-Lugo, M.; Ward, J. H.; Zhang, J. 887 Physicochemical Foundations and Structural Design of Hydrogels in 888 Medicine and Biology. Annu. Rev. Biomed. Eng. 2000, 2 (1), 9-29. 889

(51) Peppas, N. A.; Hilt, J. Z.; Khademhosseini, A.; Langer, R. 890 Hydrogels in Biology and Medicine: From Molecular Principles to 891 Bionanotechnology. Adv. Mater. 2006, 18 (11), 1345-1360. 892

(52) Pedersen, J. S.; Sommer, C. Temperature Dependence of the 893 Virial Coefficients and the Chi Parameter in Semi-Dilute Solutions of 894 PEG. Prog. Colloid Polym. Sci. 2005, 130, 70-78.

(53) Hays, G. C.; Bastian, T.; Doyle, T. K.; Fossette, S.; Gleiss, A. C.; 896 Gravenor, M. B.; Hobson, V.J.; Humphries, N. E.; Lilley, M. K.S.; Pade, 897 N. G.; et al. High Activity and Levy Searches: Jellyfish Can Search the 898 
899 Water Column like Fish. Proc. R. Soc. London, Ser. B 2012, 279 (1728), $900465-473$.

901 (54) Göröcs, Z.; Rivenson, Y.; Ceylan Koydemir, H.; Tseng, D.; Troy, 902 T. L.; Demas, V.; Ozcan, A. Quantitative Fluorescence Sensing 903 Through Highly Autofluorescent, Scattering, and Absorbing Media 904 Using Mobile Microscopy. ACS Nano 2016, 10 (9), 8989-8999.

905 (55) Lim, C.; Leamaster, B.; Brock, J. A. Riboflavin Requirement of 906 Fingerling Red Hybrid Tilapia Grown in Seawater. J. World Aquacult. 907 Soc. 1993, 24 (4), 451-458.

908 (56) Petteys, B. J.; Frank, E. L. Rapid Determination of Vitamin B2 909 (Riboflavin) in Plasma by HPLC. Clin. Chim. Acta 2011, 412 (1), 3891043.

911 (57) Yao, Y.; Yonezawa, A.; Yoshimatsu, H.; Omura, T.; Masuda, S.; 912 Matsubara, K. Involvement of Riboflavin Transporter RFVT2/Slc52a2 913 in Hepatic Homeostasis of Riboflavin in Mice. Eur. J. Pharmacol. 2013, 914714 (1), 281-287.

915 (58) Bashkatov, A. N.; Genina, E. A.; Kochubey, V. I.; Tuchin, V. V. 916 Optical Properties of Human Skin, Subcutaneous and Mucous Tissues 917 in the Wavelength Range from 400 to 2000 Nm. J. Phys. D: Appl. Phys. 918 2005, 38 (15), 2543-2555.

919 (59) Smith, A. M.; Mancini, M. C.; Nie, S. Bioimaging: Second 920 Window for in Vivo Imaging. Nat. Nanotechnol. 2009, 4, 710-711.

921 (60) Welsher, K.; Liu, Z.; Sherlock, S. P.; Robinson, J. T.; Chen, Z.; 922 Daranciang, D.; Dai, H. A Route to Brightly Fluorescent Carbon 923 Nanotubes for Near-Infrared Imaging in Mice. Nat. Nanotechnol. 2009, 9244 (11), 773-780.

925 (61) Luo, S.; Zhang, E.; Su, Y.; Cheng, T.; Shi, C. A Review of NIR 926 Dyes in Cancer Targeting and Imaging. Biomaterials 2011, 32 (29), $9277127-7138$.

928 (62) Iverson, N. M.; Bisker, G.; Farias, E.; Ivanov, V.; Ahn, J.; Wogan, 929 G. N.; Strano, M. S. Quantitative Tissue Spectroscopy of near Infrared 930 Fluorescent Nanosensor Implants. J. Biomed. Nanotechnol. 2016, 12 931 (5), 1035-1047.

932 (63) Hawkes, J. W. The Structure of Fish Skin. Cell Tissue Res. 1974, 933149 (713), 147-158.

934 (64) Jordan, T. M.; Partridge, J. C.; Roberts, N. W. Non-Polarizing 935 Broadband Multilayer Reflectors in Fish. Nat. Photonics 2012, 6 (11), $936759-763$.

937 (65) Spotila, J. R. Sea Turtles: A Complete Guide to Their Biology, 938 Behavior, and Conservation; Johns Hopkins University Press: Baltimore, 9392004.

940 (66) Ng, K.-C. C.; Ooi, A.-C. P. A Review of Fish Taxonomy 941 Conventions and Species Identification Techniques. J. Surv. Fish. Sci. 942 2017, 4 (1), 54-93.

943 (67) Human, B. A.; Owen, E. P.; Compagno, L. J. V.; Harley, E. H. 944 Testing Morphologically Based Phylogenetic Theories within the 945 Cartilaginous Fishes with Molecular Data, with Special Reference to the 946 Catshark Family (Chondrichthyes; Scyliorhinidae) and the Interrela947 tionships within Them. Mol. Phylogenet. Evol. 2006, 39 (2), 384-391. 948 (68) Kosoglu, M. A.; Hood, R. L.; Chen, Y.; Xu, Y.; Rylander, M. N.; 949 Rylander, C. G. Fiber Optic Microneedles for Transdermal Light 950 Delivery: Ex Vivo Porcine Skin Penetration Experiments. J. Biomech. 951 Eng. 2010, 132 (9), 91014-91017.

952 (69) Matzeu, G.; Florea, L.; Diamond, D. Advances in Wearable 953 Chemical Sensor Design for Monitoring Biological Fluids. Sens. 954 Actuators, B 2015, 211, 403-418.

955 (70) Allaby, M. Carcharhinidae. In A Dictionary of Zoology; Oxford 956 University Press, 2009.

957 (71) Jacques, S. L. Optical Properties of Biological Tissues: A Review. 958 Phys. Med. Biol. 2013, 58 (11), R37-R61.

959 (72) Anderson, R. R.; Parrish, J. A. The Optics of Human Skin. J. 960 Invest. Dermatol. 1981, 77 (1), 13-19.

961 (73) Nassar, J. M.; Khan, S. M.; Velling, S. J.; Diaz-Gaxiola, A.; Shaikh, 962 S. F.; Geraldi, N. R.; Torres Sevilla, G. A.; Duarte, C. M.; Hussain, M. 963 M. Compliant Lightweight Non-Invasive Standalone "Marine Skin" 964 Tagging System. npj Flex. Electron. 2018, 2 (1), 13.

965 (74) Ando, A.; Hatori, M.; Hagiwara, Y.; Isefuku, S.; Itoi, E. Imaging 966 Features of Foreign Body Granuloma in the Lower Extremities
Mimicking a Soft Tissue Neoplasm. Upsala J. Med. Sci. 2009, 114 (1), 967 $46-51$.

(75) Anderson, J. M.; Rodriguez, A.; Chang, D. T. Foreign Body 969 Reaction to Biomaterials. Semin. Immunol. 2008, 20 (2), 86-100. 970

(76) Adelman, J. S.; Martin, L. B. Vertebrate Sickness Behaviors: 971 Adaptive and Integrated Neuroendocrine Immune Responses. Integr. 972 Comp. Biol. 2009, 49 (3), 202-214.

(77) Ashley, P. J.; Sneddon, L. U. Pain and Fear in Fish. In Fish 974 Welfare; Branson, E. J., Ed.; Blackwell, 2008; 49-77. 975

(78) Stoskopf, M. K. Biology and Management of Laboratory Fishes. 976 In Laboratory Animal Medicine, Anderson, L. C.; Otto, G. M.; Pritchett- 977 Corning, K. R.; Whary, M. T. B. T., Eds.; Academic Press: Boston, 978 2015; pp 1063-1086. 